\title{
Gravity with de Sitter and unitary tangent groups
}

\author{
Ali H. Chamseddine ${ }^{a, c}$ and Viatcheslav Mukhanov ${ }^{b, d}$ \\ ${ }^{a}$ Physics Department, American University of Beirut, Lebanon \\ ${ }^{b}$ Theoretical Physics, Ludwig Maxmillians University, \\ Theresienstr. 37, 80333 Munich, Germany \\ ${ }^{c}$ I.H.E.S. F-91440 Bures-sur-Yvette, France \\ ${ }^{d}$ Department of Physics, New York University, NY 10003, U.S.A. \\ E-mail: achamseddine@gmail.com, \\ mukhanov@theorie.physik. uni-muenchen.de
}

ABSTRACT: Einstein Gravity can be formulated as a gauge theory with the tangent space respecting the Lorentz symmetry. In this paper we show that the dimension of the tangent space can be larger than the dimension of the manifold and by requiring the invariance of the theory with respect to $5 \mathrm{~d}$ Lorentz group (de Sitter group) Einstein theory is reproduced unambiguously. The other possibility is to have unitary symmetry on a complex tangent space of the same dimension as the manifold. In this case the resultant theory is EinsteinStrauss Hermitian gravity. The tangent group is important for matter couplings. We show that in the de Sitter case the 4 dimensional space time vector and scalar are naturally unified by a hidden symmetry being components of a $5 \mathrm{~d}$ vector in the tangent space. With a de Sitter tangent group spinors can exist only when they are made complex or taken in doublets in a way similar to $\mathrm{N}=2$ supersymmetry.

Keywords: Gauge Symmetry, Classical Theories of Gravity, Space-Time Symmetries

ARXIV EPRINT: 1002.0541 


\section{Contents}

1 Introduction 1

2 Gravity with de Sitter tangent group 2

$\begin{array}{lll}3 & \text { Matter couplings } & 7\end{array}$

4 Complex gravity and unitary $U(1, d-1)$ tangent group 9

5 Conclusions $\quad 14$

$\begin{array}{ll}\text { A The Poincare limit and 3d CS gravity } & 14\end{array}$

\section{Introduction}

The experimental evidence that Lorentz symmetry is preserved for effective fourdimensional theories is overwhelming. In curved space-time this Lorentz symmetry is realized as a local symmetry of the tangent manifold [1, 2]. Moreover, to incorporate spinors in general relativity, we are forced to consider this local symmetry because there are no spinor representations of the diffeomorphism group. Usually the dimension of the tangent space is taken to be equal to the dimension of the curved manifold and then the Lorentz symmetry is simply a manifestation of the equivalence principle, which is valid in torsion-free theories. General relativity could then be formulated as a gauge theory of the Lorentz group where the gauge fields are the spin-connection. In reality one can search for all possible tangent groups in $d$-dimensional space-time [3]. In this paper we will investigate whether it is possible to have a larger group of symmetry in the tangent space and still unambiguously reproduce general relativity. We will show in section 2 , that this is indeed possible by taking the tangent space to be real with de Sitter group symmetry. The de Sitter gauge invariant action which is linear in curvature is shown to be identical to Einstein gravity, provided that metricity condition is imposed on the spin and affine connections. In section 3 we consider matter interactions of gravity with the de Sitter group as the tangent group. We then, in section 4, consider a complex tangent space and show that the relevant symmetry in this case is the unitary symmetry. The resultant theory is the Einstein-Strauss theory. Section 5 is the conclusion. An appendix treats the special limit of Poincare symmetry, and examines the relation of our new formalism in three dimensions with Witten's formulation of Chern-Simons gravity. 


\section{Gravity with de Sitter tangent group}

Let us begin with a $d$-dimensional manifold and assume that at every point of this manifold there is a real $N$-dimensional tangent space spanned by linearly independent vectors $\mathbf{v}_{A}$, where $A=1,2 \ldots N$. Assuming that $d \leq N$, the coordinate basis vectors $\mathbf{e}_{\alpha} \equiv \partial / \partial x^{\alpha}$, where $\alpha=1,2 \ldots d$, span $d$-dimensional space. Next we define the scalar product in the tangent space and take the vectors $\mathbf{v}_{A}$ to be orthonormal ${ }^{1}$

$$
\mathbf{v}_{A} \cdot \mathbf{v}_{B}=\eta_{A B}
$$

where $\eta_{A B}$ is Minkowski matrix. The Lorentz transformations

$$
\tilde{\mathbf{v}}_{A}=\Lambda_{A}^{B} \mathbf{v}_{B}, \quad \Lambda_{A}^{C} \eta_{C D} \Lambda_{A}^{D}=\eta_{A B}
$$

preserve the orthogonality of the vielbein, $\tilde{\mathbf{v}}_{A} \cdot \tilde{\mathbf{v}}_{B}=\eta_{A B}$. The scalar product of coordinate basis vectors then induces the metric in $d$-dimensional manifold

$$
\mathbf{e}_{\alpha} \cdot \mathbf{e}_{\beta}=g_{\alpha \beta}\left(x^{\gamma}\right)
$$

Expanding $\mathbf{e}_{\alpha}$ in $\mathbf{v}_{A}$-basis

$$
\mathbf{e}_{\alpha}=e_{\alpha}^{B} \mathbf{v}_{B}
$$

and substituting in (2.3) we obtain the following expression for the metric $g_{\alpha \beta}$

$$
g_{\alpha \beta}=e_{\alpha}^{A} e_{\beta}^{B} \eta_{A B}
$$

in terms of components. Tangent space indices are raised and lowered with the Minkowski metric, thus

$$
e_{A \alpha}=\eta_{A B} e_{\alpha}^{B}=\left(\mathbf{v}_{A} \cdot \mathbf{e}_{\alpha}\right),
$$

and $\eta^{A B}$ is inverse to Minkowski matrix $\eta_{A B}$. Next we consider parallel transport on the manifold relating vectors in "nearby" tangent spaces. The affine and spin connections determining the rules for parallel transport of the coordinate basis vectors and vielbein are defined via

$$
\nabla_{\mathbf{e}_{\beta}} \mathbf{e}_{\alpha} \equiv \nabla_{\beta} \mathbf{e}_{\alpha}=\Gamma_{\alpha \beta}^{\nu} \mathbf{e}_{\nu}, \quad \nabla_{\beta} \mathbf{v}_{A}=-\omega_{\beta A}^{B} \mathbf{v}_{B},
$$

where $\nabla_{\beta}$ is the derivative defining the rate of change of vectors along a basis vector $\mathbf{e}_{\beta}$. When applied to a scalar function $f$ this derivative acts as a partial derivative with respect to the appropriate coordinates, that is, $\nabla_{\beta} f=\partial f / \partial x^{\beta}$. Notice that $\eta_{A B}$ and $g_{\alpha \beta}$ as defined in (2.1) and (2.3) are the sets of scalar functions and, hence, $\nabla_{\beta} \eta_{A B}=0$, $\nabla_{\gamma} g_{\alpha \beta}=\partial g_{\alpha \beta} / \partial x^{\gamma} \equiv \partial_{\gamma} g_{\alpha \beta}$

Given $\eta_{A B}, g_{\alpha \beta}$ and $e_{\alpha}^{A}$ we derive the consistency (metricity) conditions for the connections by taking derivative of equations (2.1), (2.3) and (2.6). In particular, we obtain

$$
\left(\nabla_{\alpha} \mathbf{v}_{A}\right) \cdot \mathbf{v}_{B}+\mathbf{v}_{A} \cdot\left(\nabla_{\alpha} \mathbf{v}_{B}\right)=-\omega_{\alpha A B}-\omega_{\alpha B A}=\nabla_{\alpha} \eta_{A B}=0
$$

\footnotetext{
${ }^{1}$ We use the notation and methods of Misner, Thorne and Wheeler ([4]), in particular sections 9 and 10.
} 
that is, the spin connection should be antisymmetric with respect to tangent space indices, $\omega_{\alpha A B}=-\omega_{\alpha B A}$. Applying the derivative $\nabla_{\gamma}$ to (2.3) gives

$$
\Gamma_{\alpha \gamma}^{\nu} g_{\nu \beta}+\Gamma_{\beta \gamma}^{\nu} g_{\alpha \nu}=\partial_{\gamma} g_{\alpha \beta}
$$

Assuming that torsion is absent, $\Gamma_{\alpha \beta}^{\nu}=\Gamma_{\beta \alpha}^{\nu}$, these equations are solved unambiguously, giving the well known result

$$
\Gamma_{\alpha \beta}^{\gamma}=\frac{1}{2} g^{\gamma \sigma}\left(g_{\alpha \sigma, \beta}+g_{\sigma \beta, \alpha}-g_{\alpha \beta, \sigma}\right),
$$

where $g^{\gamma \sigma}$ is inverse to $g_{\alpha \beta}$, that is, $g^{\alpha \sigma} g_{\sigma \beta}=\delta_{\beta}^{\alpha}$. We would like to stress that affine connections are determined unambiguously irrespective of the group of tangent space. Finally, from (2.6) we obtain

$$
\partial_{\beta} e_{A \alpha}=-\omega_{\beta A}^{B} e_{B \alpha}+\Gamma_{\alpha \beta}^{\nu} e_{A \nu}
$$

Let us find when these equations can unambiguously be solved for $\omega_{\beta B}{ }^{A}$ in terms of the soldering form $e_{\alpha}^{B}$ and metric $g_{\alpha \beta}$. The total number of components of $e_{\alpha}^{B}$ is $N d$. Given a metric $g_{\alpha \beta}$, whose derivatives determine $\Gamma_{\alpha \beta}^{\nu}$ via $(2.10)$, and hence impose $\frac{1}{2} d^{2}(d+1)$ constraints on $\partial_{\beta} e_{A \alpha}$, leaves us with $d\left(N d-\frac{1}{2} d(d+1)\right)$ independent equations (2.11) to determine $\frac{1}{2} d N(N-1)$ antisymmetric spin connections $\omega_{\beta A B}$. Note that for any $N$ and $d$ the number of equations can never exceed the number of independent $\omega_{\beta A B}$ to be determined, and hence for any dimension of tangent space the system of equations is not overdetermined. However, the spin connection is unambiguously determined only if the number of equations is equal to the number of its unknown components:

$$
d\left(N d-\frac{1}{2} d(d+1)\right)=\frac{1}{2} d N(N-1) .
$$

The only solutions of this equation are $N=d$ and $N=d+1$. The first case is well known and thus we shall concentrate on the second case which corresponds to the larger symmetry group $S O(1, d)$ of the tangent space. In the case of a four-dimensional manifold the tangent space is five dimensional. The metric in $5 \mathrm{~d}$ tangent space can then be taken either to be $\eta_{A B}=\operatorname{diag}(1,-1,-1,-1,-1)$ or $\eta_{A B}=\operatorname{diag}(1,1,-1,-1,-1)$. In the first case the gauge group is $5 \mathrm{~d}$ Lorentz group $S O(1,4)$ which is also the group of symmetry of $4 \mathrm{~d}$ de Sitter space (de Sitter group), while in the second case the group is $S O(2,3)$ (the group of symmetry of $4 \mathrm{~d}$ anti de Sitter space). For definiteness and from here on, we consider these cases only. Note that although the consistency equations do not lead to any contradiction for an arbitrary dimension of tangent space the connections are entirely determined by the soldering form only if $N=d$ or $N=d+1$. Otherwise the spin connection is not unambiguously determined by the fundamental soldering form and the theory is not well defined.

In order to construct gauge invariant Lagrangians we need to define

$$
e_{A}^{\alpha}=g^{\alpha \gamma} e_{\gamma A}=g^{\alpha \gamma} \eta_{A B} e_{\gamma}^{B} .
$$

Rewritten in terms of $e_{A}^{\alpha}$, equation (2.11) becomes

$$
\partial_{\beta} e_{A}^{\alpha}=-\omega_{\beta A}{ }^{B} e_{B}^{\alpha}-\Gamma_{\nu \beta}^{\alpha} e_{A}^{\nu} .
$$


The soldering form $e_{A}^{\alpha}$ is inverse to $e_{\beta}^{B}$ only if the dimension of the tangent space and the dimension of the manifold match. In case of a de Sitter tangent group contraction over tangent space indices gives

$$
e_{A}^{\alpha} e_{\beta}^{A}=g^{\alpha \gamma} \eta_{A B} e_{\gamma}^{B} e_{\beta}^{A}=g^{\alpha \gamma} g_{\gamma \beta}=\delta_{\beta}^{\alpha},
$$

however, contraction over space-time indices gives

$$
e_{A}^{\alpha} e_{\alpha}^{B} \neq \delta_{B}^{A}
$$

To prove this, let us introduce the unit vector $\mathbf{n}$ orthogonal to all $\mathbf{e}_{\alpha}$, that is, $\mathbf{n} \cdot \mathbf{e}_{\alpha}=0$ and $\mathbf{n} \cdot \mathbf{n}=\varepsilon$, where $\varepsilon=-1$ or +1 for de Sitter and anti de Sitter groups correspondingly. The vectors $\mathbf{n}$ and $\mathbf{e}_{\alpha}$ form a complete basis in tangent space and therefore

$$
\mathbf{v}_{A}=v_{A}^{\alpha} \mathbf{e}_{\alpha}+n_{A} \mathbf{n} .
$$

Taking into account (2.6) we have

$$
v_{A}^{\alpha}=g^{\alpha \gamma}\left(\mathbf{v}_{A} \cdot \mathbf{e}_{\gamma}\right)=g^{\alpha \gamma} \eta_{A B} e_{\gamma}^{B}=e_{A}^{\alpha},
$$

that is, the soldering form $e_{A}^{\alpha}$ coincides with the coefficient $v_{A}^{\alpha}$ in expansion (2.16). Taking this into account one gets

$$
\eta_{A B}=\mathbf{v}_{A} \cdot \mathbf{v}_{B}=v_{A}^{\alpha} v_{B}^{\beta} g_{\alpha \beta}+\varepsilon n_{A} n_{B}=e_{A}^{\alpha} e_{\alpha B}+\varepsilon n_{A} n_{B},
$$

or after rasing the tangent space index we obtain

$$
e_{A}^{\alpha} e_{\alpha}^{B}=\delta_{A}^{B}-\varepsilon n_{A} n^{B} \equiv P_{B}^{A}
$$

where $P_{B}^{A}$ is a projection operator: $P_{C}^{A} P_{B}^{C}=P_{B}^{A}$.

The components $n_{A}$ satisfy the following relations

$$
n^{A} e_{A}^{\alpha}=0, \quad n_{A} n^{A}=\varepsilon .
$$

To prove this let us note that it follows from (2.16) that $\mathbf{v}_{A} \cdot \mathbf{n}=\varepsilon n_{A}$. Substituting here the expansion

$$
\mathbf{n}=\tilde{n}^{B} \mathbf{v}_{B}
$$

we infer that $\tilde{n}^{B}=\varepsilon n^{B}$ and hence

$$
\mathbf{n}=\varepsilon n^{B} \mathbf{v}_{B}=\varepsilon\left(n^{B} e_{B}^{\alpha} \mathbf{e}_{\alpha}+n^{B} n_{B} \mathbf{n}\right),
$$

from which (2.20) immediately follows.

In vielbein formalism the soldering form $e_{A}^{\alpha}$ is a fundamental quantity and the group of symmetry under which the theory is required to be invariant is the group of local Lorentz transformations (2.2), where $\Lambda_{A}{ }^{B}=\Lambda_{A}{ }^{B}(x)$. Under Lorentz transformation we have

$$
\tilde{\mathbf{v}}_{A}=\Lambda_{A}^{B} \mathbf{v}_{B}=\Lambda_{A}^{B}\left(e_{B}^{\alpha} \mathbf{e}_{\alpha}+n_{B} \mathbf{n}\right)=\tilde{e}_{A}^{\alpha} \mathbf{e}_{\alpha}+\tilde{n}_{A} \mathbf{n},
$$


and hence

$$
e_{A}^{\alpha} \rightarrow \tilde{e}_{A}^{\alpha}=\Lambda_{A}^{B} e_{B}^{\alpha}
$$

The transformation law for the spin connection follows from its definition:

$$
\tilde{\omega}_{\beta A}{ }^{B} \tilde{\mathbf{v}}_{B}=-\nabla_{\beta} \tilde{\mathbf{v}}_{A}
$$

Substituting $\tilde{\mathbf{v}}_{B}=\Lambda_{A}{ }^{C} \mathbf{v}_{C}$ and taking into account (2.7) we infer that

$$
\omega_{\mu A}^{B} \rightarrow \tilde{\omega}_{\mu A}^{B}=\left(\Lambda \omega_{\mu} \Lambda^{-1}\right)_{A}^{B}+\left(\Lambda \partial_{\mu} \Lambda^{-1}\right)_{A}^{B},
$$

where $\Lambda$ and $\Lambda^{-1}$ are the matrices corresponding to Lorentz transformation and its inverse. Up to this point, we have considered only vector representations of the Lorentz group. In general,

$$
\Lambda=\exp \left(\lambda^{A B} J_{A B}\right)
$$

where $J_{A B}$ are corresponding generators of the Lie algebra which satisfy the commutation relations

$$
\left[J_{A B}, J_{C D}\right]=\frac{1}{2}\left(\eta_{B C} J_{A D}-\eta_{A C} J_{B D}-\eta_{B D} J_{A C}+\eta_{A D} J_{B C}\right)
$$

Consider spinors $\psi$ which transforms according to

$$
\psi \rightarrow \exp \left(\frac{1}{4} \lambda^{A B} \Gamma_{A B}\right) \psi,
$$

where $\Gamma_{A B}=\frac{1}{2}\left(\Gamma_{A} \Gamma_{B}-\Gamma_{B} \Gamma_{A}\right)$ are generators of the Lie algebra in the spinor representation and $\Gamma_{A}$ are $d+1$ Dirac matrices satisfying

$$
\left\{\Gamma^{A}, \Gamma^{B}\right\}=2 \eta^{A B}, \quad \Gamma^{\dagger A}=\Gamma^{0} \Gamma^{A} \Gamma^{0} .
$$

We note that the signature of $\eta^{A B}$ does not play any significant role in the derivations that follow, and thus our results holds equally well for both de Sitter and anti de Sitter tangent groups. The Dirac action

$$
\int d^{4} x \sqrt{g} \bar{\psi} i \Gamma^{C} e_{C}^{\alpha} D_{\alpha} \psi
$$

where

$$
D_{\alpha} \equiv \partial_{\alpha}+\frac{1}{4} \omega_{\alpha}^{A B} \Gamma_{A B}
$$

is invariant under gauge transformations (2.24), (2.25) and (2.28). This action is real, thanks to the metricity conditions (2.13).

Next one constructs the curvature of the connection $D_{\mu}$ defined by $[5,6]$

$$
\left[D_{\mu}, D_{\nu}\right]=\frac{1}{4} R_{\mu \nu}^{A B} \Gamma_{A B},
$$

where

$$
R_{\mu \nu}^{A B}(\omega)=\partial_{\mu} \omega_{\nu}^{A B}-\partial_{\nu} \omega_{\mu}^{A B}+\omega_{\mu}^{A C} \omega_{\nu C}{ }^{B}-\omega_{\nu}{ }^{A C} \omega_{\mu C}{ }^{B} .
$$

This curvature transforms as

$$
\left(R_{\mu \nu}\right)_{A}^{B} \rightarrow\left(\Lambda R \Lambda^{-1}\right)_{A}^{B},
$$


and hence

$$
R(\omega)=e_{A}^{\mu} R_{\mu \nu}^{A B}(\omega) e_{B}^{\nu},
$$

is invariant under local gauge transformations. The gauge invariant action is then given by

$$
S=-\frac{1}{2 \kappa^{2}} \int d^{4} x \sqrt{g} R(\omega)
$$

Although this action appears to depend on the non-diagonal $e_{A}^{\mu}$, it is a function of $g_{\mu \nu}$ only.

To prove this we first find how the tangent space covariant derivative acts on the components of a vector $\mathbf{l}=l^{C} \mathbf{v}_{C}$. Using spinor representation for the vector we have

$$
D_{\nu}\left(l^{D} \Gamma_{D}\right)=\partial_{\nu} l^{D} \Gamma_{D}+\frac{1}{4} \omega_{\nu}^{B C}\left[\Gamma_{B C}, \Gamma_{D}\right] l^{D} .
$$

Taking into account the commutation relation $\left[\Gamma_{B C}, \Gamma_{D}\right]=2\left(\eta_{C D} \Gamma_{B}-\eta_{B D} \Gamma_{C}\right)$ one gets

$$
D_{\nu}\left(l^{D} \Gamma_{D}\right)=\left(\partial_{\nu} l^{D}+\omega_{\nu}^{D} l^{C}\right) \Gamma_{D}
$$

and hence we deduce

$$
D_{\nu} l^{D}=\partial_{\nu} l^{D}+\omega_{\nu}{ }^{D} l^{C}
$$

In particular, it follows that

$$
D_{\nu} e^{\rho A}=\partial_{\nu} e^{\rho A}+\omega_{\nu}{ }^{A} e^{\rho B},
$$

which in turn implies that

$$
\left[D_{\mu}, D_{\nu}\right] e^{\rho A}=R_{\mu \nu}^{A B}(\omega) e_{B}^{\rho} .
$$

On the other hand, using metricity condition (2.13), we have

$$
D_{\nu} e^{\rho A}=-\Gamma_{\nu \sigma}^{\rho} e^{\sigma A}
$$

and therefore

$$
\begin{aligned}
D_{\mu}\left(D_{\nu} e^{\rho A}\right) & =-D_{\mu}\left(\Gamma_{\nu \sigma}^{\rho} e^{\sigma A}\right)=-\left(\partial_{\mu} \Gamma_{\nu \sigma}^{\rho}\right) e^{\sigma A}-\Gamma_{\nu \sigma}^{\rho}\left(D_{\mu} e^{\sigma A}\right) \\
& =-\partial_{\mu} \Gamma_{\nu \sigma}^{\rho} e^{\sigma A}+\Gamma_{\nu \sigma}^{\rho} \Gamma_{\mu \kappa}^{\sigma} e^{\kappa A}
\end{aligned}
$$

Taking the commutator one gets

$$
\begin{aligned}
{\left[D_{\mu}, D_{\nu}\right] e^{\rho A} } & =-\left(\partial_{\mu} \Gamma_{\nu \sigma}^{\rho}-\partial_{\nu} \Gamma_{\mu \sigma}^{\rho}+\Gamma_{\mu \kappa}^{\rho} \Gamma_{\nu \sigma}^{\kappa}-\Gamma_{\nu \kappa}^{\rho} \Gamma_{\mu \sigma}^{\kappa}\right) e^{\sigma A} \\
& =-R_{\sigma \mu \nu}^{\rho}(\Gamma) e^{\sigma A}
\end{aligned}
$$

Comparing this result with (2.41) we arrive at the identity

$$
R_{\mu \nu}^{A B}(\omega) e_{B}^{\rho}=-R_{\sigma \mu \nu}^{\rho}(\Gamma) e^{\sigma A},
$$

which in turn leads to

$$
\begin{aligned}
R(\omega) & =e_{A}^{\mu} R_{\mu \nu}^{A B}(\omega) e_{B}^{\nu}=-R_{\sigma \mu \nu}^{\nu}(\Gamma) e^{\sigma A} e_{A}^{\mu} \\
& =R_{\sigma \nu \mu}^{\nu}(\Gamma) g^{\sigma \mu}=R(\Gamma) .
\end{aligned}
$$


This completes the proof that the gauge invariant action (2.36) is equivalent to Einstein action and involves only those combinations of $e_{A}^{\mu}$ which reduce to the metric $g_{\mu \nu}$. The remaining $\frac{1}{2} d(d+1)$ independent combinations of $e_{A}^{\mu}$ components represent the $\frac{1}{2} d(d+1)$ gauge degrees of freedom associated with $S O(1, d)$. Thus, we conclude that it is possible to formulate Einstein gravity as a gauge invariant theory with the tangent group being de Sitter or anti de Sitter.

We would like to stress that in proving identity (2.46) we never (and could not) assume that the soldering form $e_{A}^{\mu}$ has an inverse and, moreover, this result is valid for an arbitrary dimension of tangent space. However, as it was noticed above the theory is well defined only if $N=d$ or $N=d+1$. We could also consider a gauge invariant action involving higher order curvature invariants. One can show that even in this case the action depends only on the metric $g_{\mu \nu}$. To give an example consider all possible terms which are of second order in curvature

$$
R_{\mu \nu}^{A B} R_{\rho \sigma}^{C D}\left(c_{1} e_{A}^{\mu} e_{B}^{\nu} e_{C}^{\rho} e_{D}^{\sigma}+c_{2} e_{A}^{\mu} e_{C}^{\nu} e_{D}^{\rho} e_{B}^{\sigma}+c_{3} e_{C}^{\mu} e_{D}^{\nu} e_{A}^{\rho} e_{B}^{\sigma}\right)
$$

because other terms are related to these three by symmetry. The first term is identical to $R^{2}(\Gamma)$, while for the second term we have

$$
R_{\mu \nu}^{A B}(\omega) e_{A}^{\mu} e_{B}^{\sigma} R_{\rho \sigma}^{C D}(\omega) e_{C}^{\nu} e_{D}^{\rho}=g^{\mu \kappa} R_{\kappa \mu \nu}^{\sigma}(\Gamma) g^{\nu \lambda} R_{\lambda \rho \sigma}^{\rho}(\Gamma) .
$$

after using the identity (2.45) twice. Similarly, the third term gives

$$
R_{\mu \nu}^{A B}(\omega) e_{A}^{\rho} e_{B}^{\sigma} R_{\rho \sigma}^{C D}(\omega) e_{C}^{\mu} e_{D}^{\nu}=g^{\kappa \rho} R_{\kappa \mu \nu}^{\sigma}(\Gamma) g^{\mu \lambda} R_{\lambda \rho \sigma}^{\nu}(\Gamma),
$$

which proves that the most general action which is second order in spin-connection curvature is identical to the one that depends on affine-connection curvature.

\section{Matter couplings}

We have seen that gravity is insensitive to the gauge group of the tangent space. In this section we will show that, to the contrary, matter "feels" the tangent space group. Let us consider the matter couplings in the case of de Sitter tangent group. In this case the fundamental spinors, vectors and tensors are defined as representations of the 5d Lorentz group of tangent space, and their Lagrangians must be invariant with respect to de Sitter symmetry. In vierbein formulation of gravity, we can exchange space-time tensors with Lorentz tensors. This is no longer valid for de Sitter tangent group because in this case the vielbein $e_{A}^{\mu}$ is not invertible and, for example, a vector in the tangent space is not equivalent to a space-time vector. In fact as we will show now the $5 \mathrm{~d}$ de Sitter vector is equivalent to $4 \mathrm{~d}$ space time vector and real space time scalar. Therefore, de Sitter tangent space "unifies" $4 \mathrm{~d}$ vectors and scalars.

Let us consider a 5 d vector $\mathbf{H}$, which can be expanded in terms of components as (see $(2.16),(2.17))$ :

$$
\mathbf{H}=H^{A} \mathbf{v}_{A}=H^{A} e_{A}^{\alpha} \mathbf{e}_{\alpha}+H^{A} n_{A} \mathbf{n}=H^{\alpha} \mathbf{e}_{\alpha}+\phi \mathbf{n},
$$


where

$$
H^{\alpha}=H^{A} e_{A}^{\alpha}, \quad \phi=H^{A} n_{A},
$$

are the components of a $4 \mathrm{~d}$ vector and a scalar, respectively. Multiplying the first equation by $e_{\alpha}^{B}$ and taking into account (2.19) we derive

$$
H^{B}=H^{\alpha} e_{\alpha}^{B}+\varepsilon \phi n^{B}
$$

since $e_{\alpha}^{B} n_{B}=0$ and $n^{A} n_{A}=\varepsilon$ (see (2.20)) it follows from here that

$$
H^{B} H_{B}=g_{\alpha \beta} H^{\alpha} H^{\beta}+\varepsilon \phi^{2} .
$$

Let us construct the curvature of $H_{A}$

$$
F_{A B}=D_{A} H_{B}-D_{B} H_{A},
$$

where $D_{A} \equiv e_{A}^{\alpha} D_{\alpha}$ and $D_{\alpha}$ is covariant derivative with respect to tangent space vector indices (see (2.39)); therefore, the components with only space time indices are scalars with respect to this derivative, for example, $D_{\alpha} H^{\beta}=\partial_{\alpha} H^{\beta}$. Taking this into account and using decomposition (3.3) we find

$$
D_{A} H^{B}=e_{A}^{\beta} e_{\alpha}^{B} \partial_{\beta} H^{\alpha}+e_{A}^{\beta} H^{\alpha} D_{\beta} e_{\alpha}^{B}+\varepsilon e_{A}^{\beta} n^{B} \partial_{\beta} \phi+\varepsilon e_{A}^{\beta} \phi D_{\beta} n^{B} .
$$

The last term here is equal to zero. In fact, using the definition (2.7) we have

$$
\partial_{\beta} n_{A}=\varepsilon \nabla_{\beta}\left(\mathbf{v}_{A} \cdot \mathbf{n}\right)=-\omega_{\beta A}{ }^{B} n_{B}+\varepsilon \mathbf{v}_{A} \cdot \nabla_{\beta} \mathbf{n},
$$

and hence $D_{\beta} n_{A}=-\varepsilon \mathbf{v}_{A} \cdot \nabla_{\beta} \mathbf{n}$. In turn, one can immediately conclude from $\nabla_{\beta}\left(\mathbf{e}_{\alpha} \cdot \mathbf{n}\right)=0$ and $\nabla_{\beta}(\mathbf{n} \cdot \mathbf{n})=0$ that $\nabla_{\beta} \mathbf{n}=0$ and therefore $D_{\beta} n_{A}=0$. Using metricity condition (2.42) to express $D_{\beta} e_{\alpha}^{B}$ in terms of $\Gamma_{\nu \sigma}^{\rho}$ and interchanging indices we then find

$$
F_{A B}=e_{A}^{\beta} e_{B}^{\alpha}\left(\partial_{\beta} H_{\alpha}-\partial_{\alpha} H_{\beta}\right)+\varepsilon\left(e_{A}^{\beta} n_{B}-e_{B}^{\beta} n_{A}\right) \partial_{\beta} \phi .
$$

Note that $F_{A B}$ is invariant under the $U(1)$ gauge transformation

$$
H_{A} \rightarrow H_{A}+e_{A}^{\alpha} \partial_{\alpha} \Lambda
$$

which in terms of the space time components become $H_{\alpha} \rightarrow H_{\alpha}+\partial_{\alpha} \Lambda, \phi \rightarrow \phi$. Squaring (3.8) we will find the gauge invariant Lagrangian density for the massless vector field

$$
L=-\frac{1}{4} F_{A B} F^{A B}=-\frac{1}{4} F_{\alpha \beta} F^{\alpha \beta}-\frac{1}{2} \varepsilon \partial_{\alpha} \phi \partial^{\alpha} \phi,
$$

where

$$
F_{\alpha \beta}=\partial_{\alpha} H_{\beta}-\partial_{\beta} H_{\alpha} .
$$

Notice that we get the correct sign for the kinetic energy of the scalar field $\phi$ only in the case of de Sitter group $(\varepsilon=-1)$ while for anti de Sitter group $\varepsilon=1$ we get a ghost. We deduce that the formulation of gravity where the tangent group is $S O(1, d)$ instead of 
$S O(1, d-1)$ unifies spins zero and spin one in one vector field. If we add to the Lagrangian the term (3.4) both fields acquire the same mass.

We now turn to spinors. Because they should respect 5 d tangent Lorentz group it is well known that neither Majorana or Weyl conditions can be imposed on them [7]. Thus the spinors $\psi$ must be Dirac spinors. The Dirac action in this case is

$$
\int \sqrt{g} d^{4} x\left(i \bar{\psi} \Gamma^{A} D_{A} \psi-i \overline{D_{A} \psi} \Gamma^{A} \psi\right)
$$

The spinors do feel the full $S O(1,4)$ local symmetry. This seems to be a very strong constraint as it implies that chiral spinors cannot exist if the tangent group is $S O(1,4)$. This is similar to the situation in case of supersymmetry in five dimensions $[8,9]$, or $N=2$ supersymmetry. There, it was shown that it is possible to generalize the Majorana condition by taking a doublet of spinors [7]. The conclusion we must draw is then that the $S O(1,4)$ tangent group implies that spinors must be treated in the same way as in $N=2$ supersymmetry. To couple the spinors to vectors, some gauge symmetry must be introduced. As an example, let us assume the existence of a $U(1)$ gauge symmetry. In this case the covariant derivative $D_{A} \psi$ becomes

$$
D_{A} \psi=\left(e_{A}^{\mu}\left(\partial_{\mu}+\frac{1}{4} \omega_{\mu}^{A B} \Gamma_{A B}\right)+i H_{A}\right) \psi,
$$

which shows that the spinors exist in a unified interactions with both a scalar and a vector field, as was seen in the decomposition of the vector $H_{A}$ into a vector $H_{\mu}$ and a scalar $\phi$.

\section{Complex gravity and unitary $U(1, d-1)$ tangent group}

As a tangent space one can also consider a complex vector space with Hermitian scalar product satisfying

$$
(\mathbf{v}, \mathbf{u})=(\mathbf{u}, \mathbf{v})^{*}, \quad(\mathbf{v}, \alpha \mathbf{u})=\alpha(\mathbf{v}, \mathbf{u}),
$$

where $\alpha$ is a complex number. It follows from here that $(\alpha \mathbf{v}, \mathbf{u})=\alpha^{*}(\mathbf{v}, \mathbf{u})$. As before let us introduce in this space the orthonormal basis $\mathbf{v}_{A}(A=1, \ldots N)$ :

$$
\left(\mathbf{v}_{A}, \mathbf{v}_{B}\right)=\eta_{A B}
$$

The condition of orthogonality is preserved under $U(1, N-1)$ transformations

$$
\tilde{\mathbf{v}}_{A}=U_{A}^{C} \mathbf{v}_{C}, \quad U_{A}^{C} \eta_{C D}\left(U_{A}^{D}\right)^{*}=\eta_{A B}
$$

For generality let us first consider the complex coordinate basis vectors $\mathbf{e}_{\alpha}(\alpha=1, \ldots d)$ in $d$-dimensional manifold and show that in this case we obtain the Hermitian theory of gravity as formulated by Einstein and Strauss [10, 11]. Later on we will show that this theory can be consistently truncated to General Relativity while preserving the unitary structure of the tangent space.

Assuming that $N \geq d$ we can expand the coordinate basis vectors in terms of vielbein vectors, $\mathbf{e}_{\alpha}=e_{\alpha}^{A} \mathbf{v}_{A}$, and then the metric on the manifold can be expressed as

$$
g_{\alpha \beta} \equiv\left(\mathbf{e}_{\alpha}, \mathbf{e}_{\beta}\right)=e_{\alpha}^{A} e_{\beta}^{B *} \eta_{A B} .
$$


This metric is Hermitian

$$
g_{\alpha \beta}=\left(\mathbf{e}_{\alpha}, \mathbf{e}_{\beta}\right)=\left(\mathbf{e}_{\beta}, \mathbf{e}_{\alpha}\right)^{*}=g_{\beta \alpha}^{*} .
$$

In the case under consideration the affine and spin connections are defined exactly as in (2.7). Taking derivative of (4.4) and using definition in (2.7) we obtain

$$
\partial_{\gamma} g_{\alpha \beta}=\left(\nabla_{\gamma} \mathbf{e}_{\alpha}, \mathbf{e}_{\beta}\right)+\left(\mathbf{e}_{\alpha}, \nabla_{\gamma} \mathbf{e}_{\beta}\right)=\Gamma_{\alpha \gamma}^{\nu *} g_{\nu \beta}+\Gamma_{\beta \gamma}^{\nu} g_{\alpha \nu}
$$

These $d^{3}$ equations can be solved unambiguously for $\Gamma_{\kappa \rho}^{\mu}$ in terms of metric $g_{\alpha \beta}$ only if we impose the hermiticity condition

$$
\Gamma_{\rho \mu}^{\nu *}=\Gamma_{\mu \rho}^{\nu},
$$

which leaves us with $d^{3}$ components to be determined. Unlike the real case equations (4.6) can be solved only perturbatively. They were first imposed by Einstein in his formulation of Hermitian gravity which he referred to as the "+-" condition [10-12]. Similar to (2.8) we derive a condition on spin connection

$$
\omega_{\alpha A}{ }^{C} \eta_{C B}=-\left(\omega_{\alpha B}^{C}\right)^{*} \eta_{C A},
$$

which leaves $N^{2} d$ independent components. Taking derivative of $\left(\mathbf{v}_{A}, \mathbf{e}_{\alpha}\right)=e_{\alpha}^{B} \eta_{A B}$ we derive the following metricity conditions

$$
\partial_{\gamma} e_{\alpha}^{A}=\omega_{\gamma B}^{A} e_{\alpha}^{B}+\Gamma_{\alpha \gamma}^{\nu} e_{\nu}^{A} .
$$

Taking into account that $d^{3}$ equations (4.5) determine $\Gamma_{\beta \gamma}^{\nu}$ through $\partial_{\gamma} e_{\alpha}^{A}$ we are left with $2 N d^{2}-d^{3}$ equations to find $N^{2} d$ independent components of $\omega_{\alpha A}{ }^{C}$. The number of equations match the number of unknown components only if $N=d$, that is, when dimension of complex tangent space coincides with the dimension of the manifold. Hence the gauge group of the tangent space can be only $U(1, d-1)[13]$. In this case we can define the soldering form $e_{B}^{\beta}$, which is inverse to $e_{\alpha}^{A}$ :

$$
e_{B}^{\alpha} e_{\alpha}^{A}=\delta_{B}^{A}, \quad e_{A}^{\alpha} e_{\beta}^{A}=\delta_{\beta}^{\alpha} .
$$

The metric with upper indices is then given by

$$
g^{\mu \nu}=e_{A}^{\mu} e_{B}^{\nu *} \eta^{A B},
$$

and it is inverse to $g_{\alpha \beta}$

$$
g_{\alpha \nu} g^{\beta \nu}=\delta_{\alpha}^{\beta} \neq g_{\alpha \nu} g^{\nu \beta} .
$$

Similar to (2.41) the curvature of the connection $\omega_{\mu A}{ }^{B}$ can be defined as

$$
\begin{aligned}
{\left[D_{\mu}, D_{\nu}\right] e_{A}^{\sigma} } & \equiv R_{\mu \nu A}{ }^{B}(\omega) e_{B}^{\sigma} \\
& =\left(\partial_{\mu} \omega_{\nu A}{ }^{B}-\partial_{\nu} \omega_{\mu A}{ }^{B}+\omega_{\mu A}{ }^{C} \omega_{\nu C}{ }^{B}-\omega_{\nu A}{ }^{C} \omega_{\mu C}{ }^{B}\right) e_{B}^{\sigma} .
\end{aligned}
$$

On the other hand, using the metricity condition, we have

$$
\begin{aligned}
{\left[D_{\mu}, D_{\nu}\right] e_{A}^{\sigma} } & =-\left(\partial_{\mu} \Gamma_{\rho \nu}^{\sigma}-\partial_{\nu} \Gamma_{\rho \mu}^{\sigma}+\Gamma_{\kappa \mu}^{\sigma} \Gamma_{\rho \nu}^{\kappa}-\Gamma_{\kappa \nu}^{\sigma} \Gamma_{\rho \mu}^{\kappa}\right) e_{A}^{\sigma} \\
& \equiv-R_{\rho \mu \nu}^{\sigma}(\Gamma) e_{A}^{\rho},
\end{aligned}
$$


and it follows from here that

$$
R_{\rho \mu \nu}^{\sigma}(\Gamma)=-e_{\rho}^{A} R_{\mu \nu A}{ }^{B}(\omega) e_{B}^{\sigma} .
$$

In particular, the scalar curvature

$$
\begin{aligned}
R(\omega) & =\eta^{A C} e_{C}^{\mu *} R_{\mu \nu A}^{B}(\omega) e_{B}^{\nu}=-\eta^{A C} e_{C}^{\mu *} R_{\rho \mu \nu}^{\nu}(\Gamma) e_{A}^{\rho} \\
& =g^{\rho \mu} R_{\rho \nu \mu}^{\nu}(\Gamma)=R(\Gamma),
\end{aligned}
$$

is $U(1, d-1)$ gauge invariant. The scalar curvature is real,

$$
R^{*}(\omega)=R(\omega)
$$

To prove this we first note the identity

$$
\left(R_{\mu \nu A}^{B}(\omega)\right)^{*}=-R_{\mu \nu C}^{D}(\omega) \eta^{C B} \eta_{D A},
$$

which follows from equation (4.12) taking into account (4.7). Using this relation together with (4.14) we obtain

$$
\begin{aligned}
\left(R_{\rho \mu \nu}^{\sigma}(\Gamma)\right)^{*} & =-e_{\rho}^{A *}\left(R_{\mu \nu A}^{B}(\omega)\right)^{*} e_{B}^{\sigma *}=e_{\rho}^{A *} R_{\mu \nu C}^{D}(\omega) \eta^{C B} \eta_{D A} e_{B}^{\sigma *} \\
& =-\eta^{C B} e_{C}^{\kappa} e_{B}^{\sigma *} R_{\kappa \mu \nu}^{\lambda}(\Gamma) \eta_{D A} e_{\lambda}^{D} e_{\rho}^{A *}=-g^{\kappa \sigma} R^{\lambda}{ }_{\kappa \mu \nu}(\Gamma) g_{\lambda \rho} .
\end{aligned}
$$

It follows from here that the tensor

$$
R_{\rho \kappa \mu \nu}(\Gamma)=R_{\kappa \mu \nu}^{\lambda}(\Gamma) g_{\lambda \rho},
$$

is antihermitian with respect to exchange of first two indices

$$
\left(R_{\kappa \rho \mu \nu}(\Gamma)\right)^{*}=-R_{\rho \kappa \mu \nu}(\Gamma),
$$

and it is antisymmetric with respect to exchange of the last two indices (see (4.13). Taking this into account we have

$$
R^{*}(\Gamma)=\left(g^{\rho \mu} g^{\nu \sigma} R_{\sigma \rho \nu \mu}\right)^{*}=g^{\mu \rho} g^{\sigma \nu} R_{\rho \sigma \mu \nu}=R(\Gamma),
$$

and because $R(\omega)=R(\Gamma)$, this completes the proof of reality of gauge invariant scalar curvature.

The identity (4.20) was not noticed by Einstein and this forced him to construct Hermitian combinations of the curvature tensor. As we see this is not necessary because one can use instead the real scalar curvature as Lagrangian density.

If we write the connection as

$$
\omega_{\mu A}{ }^{B}=\bar{\omega}_{\mu A}{ }^{B}+\frac{1}{d} \hat{\omega}_{\mu} \delta_{A}^{B},
$$

where

$$
\bar{\omega}_{\mu A}^{A}=0, \quad \hat{\omega}_{\mu}=\omega_{\mu A}^{A},
$$


the curvature splits into two pieces

$$
R_{\mu \nu A}^{B}(\omega)=R_{\mu \nu A}^{B}(\bar{\omega})+\frac{1}{d} R_{\mu \nu C}^{C}(\hat{\omega}) \delta_{A}^{B},
$$

where

$$
\begin{aligned}
& R_{\mu \nu A}{ }^{B}(\bar{\omega})=\left(\partial_{\mu} \bar{\omega}_{\nu A}{ }^{B}-\partial_{\nu} \bar{\omega}_{\mu A}{ }^{B}+\bar{\omega}_{\mu A}{ }^{C} \bar{\omega}_{\nu C}{ }^{B}-\bar{\omega}_{\nu A}{ }^{C} \bar{\omega}_{\mu C}{ }^{B}\right), \\
& R_{\mu \nu C}{ }^{C}(\omega)=\partial_{\mu} \hat{\omega}_{\nu}-\partial_{\nu} \hat{\omega}_{\mu} .
\end{aligned}
$$

It follows from here that

$$
R(\omega)=\eta^{A C} e_{C}^{\mu *} R_{\mu \nu A}^{B}(\bar{\omega}) e_{B}^{\nu}+\frac{1}{d} g^{\nu \mu} R_{\mu \nu C}^{C}(\hat{\omega})=R(\bar{\omega})+\frac{1}{d} \tilde{R}(\hat{\omega}),
$$

where $\tilde{R}=g^{\nu \mu} R_{\mu \nu A}{ }^{A}$ is another scalar curvature invariant. Therefore it can be added to the action with an arbitrary coefficient leading to the following most general gauge invariant first order action

$$
S=\int d^{4} x\left|\operatorname{det} e_{\mu}^{A}\right|(\alpha R(\bar{\omega})+\beta \tilde{R}(\hat{\omega})) .
$$

It must be stressed that we are using here a second order formalism where the field $\omega_{\mu A}{ }^{B}$ is determined by the metricity condition and not by the field equations. The best strategy to analyze this action is to solve for $\omega_{\mu A}{ }^{B}$ in a perturbative expansion in terms of $e_{\mu}^{A}$.

We can understand the above results by noting that the gauge invariant action allows to use the gauge invariance to reduce the independent components of $e_{\alpha}^{A}$ to those of $g_{\alpha \beta}$. In other words we expect that because of $U(1, d-1)$ gauge invariance, the action depends only on the metric

$$
g_{\alpha \beta}=e_{\alpha}^{A} e_{\beta}^{B *} \eta_{A B} \equiv G_{\alpha \beta}+i B_{\alpha \beta} .
$$

This theory was considered before using a first order formalism where the spin-connection was determined from the equations of motion [13]. This is possible only when the action depends quadratically on the spin-connection. However, the $U(1)$ part $\hat{\omega}$ of the $U(1, d-1)$ connection being abelian, appears linearly. This then imposes a constraint on the antisymmetric part of the metric

$$
\partial_{\alpha}\left(\left|\operatorname{det} e_{\mu}^{A}\right| B^{\alpha \beta}\right)=0
$$

which thus remains undetermined [13]. This is to be contrasted with the second order formalism where all spin-connections are determined from the metricity condition.

We arrive to an interesting case by requiring that the metric $g_{\alpha \beta}$ to be real. This is equivalent to truncating the $B_{\alpha \beta}$ field. Let

$$
e_{\alpha}^{A}=e_{\alpha(0)}^{A}+i e_{\alpha(1)}^{A},
$$

so that

$$
\begin{aligned}
G_{\alpha \beta} & =\left(e_{\alpha(0)}^{A} e_{\beta(0)}^{B}+e_{\alpha(1)}^{A} e_{\beta(1)}^{B}\right) \eta_{A B}, \\
B_{\alpha \beta} & =\left(e_{\alpha(1)}^{A} e_{\beta(0)}^{B}-e_{\alpha(0)}^{A} e_{\beta(1)}^{B}\right) \eta_{A B},
\end{aligned}
$$


Truncating $B_{\alpha \beta}$ gives $\frac{1}{2} d(d-1)$ constraints on the $2 d^{2}$ (real) fields $e_{\alpha(0)}^{A}$ and $e_{\alpha(1)}^{A}$. In this case the affine connection is also real and its $\frac{1}{2} d^{2}(d+1)$ components are Christoffel connection for the metric $G_{\alpha \beta}$. The remaining

$$
2 d^{3}-\frac{1}{2} d d(d-1)-\frac{1}{2} d^{2}(d+1)=d^{3}
$$

independent equations (4.8) are then enough to unambiguously determine $d^{3}$ components of $\omega_{\mu A}{ }^{B}$. This implies that it is possible to enlarge the tangent group to become $U(1, d-1)$ and still obtain the Einstein gravity without any modification. The coupling to matter will, however, feel the tangent group $U(1, d-1)$.

Matter coupling. When the tangent group is $U(1,3)$ then from the previous discussion it should be clear that neither the Majorana nor the Weyl condition could be imposed, except if a doublet of spinors is taken. Thus, as with the $S O(1,4)$ case we must take a Dirac spinor, or a doublet of Majorana or Weyl spinors, again as in the $N=2$ supersymmetric case. We note the isomorphism of the algebras

$$
U(1,3) \sim S O(1,5) \times S O(1,1) .
$$

It is easy to see that $U(1,3)$ has ten compact generators and six non-compact generators, while $S O(1,5)$ has ten compact generators and five non-compact generators and $S O(1,1)$ has one non-compact generator. Thus spinors in the case of unitary tangent group will exhibit conformal local symmetry.

Gravity has a universal coupling to matter. One way to classify the fields is according to their behavior under the diffeomorphism group, or equivalently under the tangent Lorentz group. A complex scalar field has the following couplings

$$
\int d^{4} x \sqrt{\operatorname{det} g} g^{\mu \nu} \partial_{\mu} \phi \partial_{\nu} \phi^{*}
$$

For a massless vector it can be easily seen that the action can be written in terms of a complex space-time vector $H_{\mu}$ with the action

$$
\int d^{4} x \sqrt{\operatorname{det} g} g^{\mu \rho} g^{\nu \sigma} F_{\mu \nu} F_{\rho \sigma}^{*} .
$$

Similarly we can treat the case of fields which are in the vector representations of the gauge group. The fermions have more complicated couplings. First, a Dirac spinor has the $U(1, d-1)$ transformation

$$
\psi \rightarrow e^{i \lambda_{B}^{A} \Gamma_{A} \Gamma^{B}} \psi
$$

where $\Gamma^{A}$ and $\Gamma_{A}$ satisfy the relations

$$
\left\{\Gamma^{A}, \Gamma^{B}\right\}=0, \quad\left\{\Gamma_{A}, \Gamma_{B}\right\}=0, \quad\left\{\Gamma^{A}, \Gamma_{B}\right\}=\delta_{B}^{A},
$$

and thus $\Gamma_{A} \Gamma^{B}$ are the generators of $U(1, d-1)$. We can define the Hermitian Dirac matrices

$$
\begin{aligned}
\gamma^{\mu} & =e_{A}^{\mu} \Gamma^{A}+e^{\mu A} \Gamma_{A}, \\
\left\{\gamma^{\mu}, \gamma^{\nu}\right\} & =g^{\mu \nu}+g^{\nu \mu},
\end{aligned}
$$


The covariant derivative is given by

$$
D_{\mu} \psi=\partial_{\mu} \psi+\omega_{\mu B}{ }^{A} \Gamma_{A} \Gamma^{B} \psi,
$$

Hermitian Dirac action is then

$$
\int d^{4} x\left|\operatorname{det} e_{\mu}^{A}\right| \bar{\psi} \gamma^{\mu} D_{\mu} \psi
$$

Therefore, Dirac spinors do couple to both the symmetric and antisymmetric components of the Hermitian metric.

\section{Conclusions}

We have shown that Einstein gravity exhibits universality when formulated as a gauge theory of tangent space group. Besides of the well known natural case when the tangent space has the same dimension as the manifold, we discovered two other possibilities for General Relativity to be reproduced and the theory still remains unambiguous. Namely, we have shown that in the four dimensional case the tangent space can be five dimensional and possess (anti) de Sitter group of symmetry. This group is important when we incorporate matter couplings to the gravitational field. As an example, we have shown that de Sitter tangent space group allows us to "unify" $4 \mathrm{~d}$ vectors and scalars which become components of the same five dimensional vector in tangent space. Even more dramatic are the consequences of the tangent space symmetry group on fermions. They become fundamentally five dimensional and neither Majorana nor Weyl conditions could be imposed on them. This situations is similar to $N=2$ supersymmetry where we are forced to generalize the Majorana condition by taking a doublet of spinors. We also would like to note that if we impose an extra $U(1)$ local symmetry in the tangent space then the spinors would exist in a unified interaction with both scalar and vector fields.

Another interesting possibility arise when we consider complex tangent space of the same dimension as the manifold. In this case the group of symmetry is the unitary group. This gives rise generically to the theory of Hermitian gravity, where the basic fields are the symmetric and antisymmetric components of the metric, which coincide with the basic fields appearing in effective open string field theory. It is interesting that this theory can be consistently truncated to Einstein gravity, while still preserving the unitary group of tangent space. In turn, this has interesting and nontrivial consequences for the coupling to matter which should respect this symmetry. In a forthcoming paper [14] we shall explore the implications of these new formulations of gravity, especially in regard to the spontaneous breakdown of these larger symmetries down to the $S O(1, d-1)$ symmetry.

\section{A The Poincare limit and 3d CS gravity}

In this appendix we examine the special case when the radius of the de Sitter tangent group becomes infinite, which corresponds to Poincare symmetry. Later we shall also investigate the correspondence with Chern-Simons gravity in three dimensions which also have de Sitter or Poincare symmetry $[15,16]$. 
The $S O(1, d)$ group generators satisfy the commutation relations

$$
\left[J_{A B}, J_{C D}\right]=-\frac{1}{2}\left(\eta_{A C} J_{B D}-\eta_{B C} J_{A D}-\eta_{A D} J_{B C}+\eta_{B D} J_{A C}\right) .
$$

Splitting the range of the index $A=a, \bar{d}$, where $a=0,1, \cdots, d-1$, and similarly for the other indices we get the usual $S O(1, d-1)$ for the $J_{a b}$, while for $J_{a \bar{d}} \equiv R P_{a}$ we have

$$
\left[P_{a}, P_{b}\right]=-\frac{1}{R^{2}} J_{a b}
$$

Thus, in the limit $R \rightarrow \infty$ the de Sitter tangent group becomes the inhomogeneous Lorentz group, i.e. $I S O(1, d-1)$ also known as the Poincare group. The covariant derivative

$$
D_{\mu}=\partial_{\mu}+\omega_{\mu}^{A B} J_{A B}
$$

implies that the field $\omega_{\mu}^{a \bar{d}}$ must be defined as $\omega_{\mu}^{a \bar{d}} \equiv \frac{1}{2 R} b_{\mu}^{a}$ so that

$$
D_{\mu}=\partial_{\mu}+\omega_{\mu}^{a b} J_{a b}+b_{\mu}^{a} P_{a},
$$

is independent of the radius $R$. The curvatures in terms of the redefined fields are

$$
\begin{aligned}
& R_{\mu \nu}^{a b}=\partial_{\mu} \omega_{\nu}^{a b}-\partial_{\nu} \omega_{\mu}^{a b}+\omega_{\mu}^{a c} \omega_{\nu c}^{b}-\omega_{\nu}^{a c} \omega_{\mu c}^{b}-\frac{1}{4 R^{2}}\left(b_{\mu}^{a} b_{\nu}^{b}-b_{\mu}^{b} b_{\nu}^{a}\right) \\
& R_{\mu \nu}^{a \bar{d}}=\frac{1}{2 R}\left(\partial_{\mu} b_{\nu}^{a}-\partial_{\mu} b_{\nu}^{a}+\omega_{\mu}^{a c} b_{\nu c}-\omega_{\nu}^{a c} b_{\mu c}\right) .
\end{aligned}
$$

The zero torsion condition on $e_{A}^{\mu}$ is consistent in the limit $R \rightarrow \infty$ if we define

$$
e_{\bar{d}}^{\mu} \equiv \frac{1}{R} c^{\mu}
$$

so that

$$
\partial_{\mu} c^{\nu}-\frac{1}{2} b_{\mu}^{a} e_{a}^{\nu}+\Gamma_{\rho \mu}^{\nu} c^{\rho}=0
$$

which allows us to calculate $b_{\mu}^{a}$ in terms of $c^{\mu}$. The field $\omega_{\mu}{ }^{a b}$ is solved from the condition

$$
\partial_{\mu} e_{a}^{\nu}+\omega_{\mu a}^{b} e_{b}^{\nu}+\frac{1}{2 R^{2}} b_{\mu}^{a} c^{\nu}+\Gamma_{\rho \mu}^{\nu} e_{a}^{\rho}=0 .
$$

Writing the gravitational action in terms of the rescaled fields, we expand $e_{A}^{\mu} R_{\mu \nu}^{A B}(\omega) e_{B}^{\nu}$ to get

$$
\begin{aligned}
& e_{a}^{\mu} e_{b}^{\nu}\left(\partial_{\mu} \omega_{\nu}^{a b}-\partial_{\nu} \omega_{\mu}^{a b}+\omega_{\mu}^{a c} \omega_{\nu c}^{b}-\omega_{\nu}^{a c} \omega_{\mu c}^{b}-\frac{1}{4 R^{2}}\left(b_{\mu}^{a} b_{\nu}^{b}-b_{\mu}^{b} b_{\nu}^{a}\right)\right) \\
& +\frac{1}{R^{2}} e_{a}^{\mu} c^{\nu}\left(\partial_{\mu} b_{\nu}^{a}-\partial_{\nu} b_{\mu}^{a}+\omega_{\mu}^{a c} b_{\nu c}-\omega_{\nu}^{a c} b_{\mu c}\right)
\end{aligned}
$$

Therefore it is clear that in the limit $R \rightarrow \infty$ the connection $\omega_{\mu}^{a b}$ coincides with the $S O(1, d-1)$ Lorentz connection and the action becomes identical to the Einstein-Hilbert action. The fields $b_{\mu}^{a}$ and $c^{\mu}$ drop out of the action. Thus in the limit of $I S O(1, d-1)$ the action is indistinguishable from the $S O(1, d-1)$ invariant action for gravity. 
For matter couplings, especially for the vector $H_{A}$, the gauge transformation is

$$
\delta H_{A}=\lambda_{A B} H^{B}, \quad \lambda_{A B}=-\lambda_{B A} .
$$

Denoting $H_{\bar{d}}=\phi$ and $\lambda_{a \bar{d}}=\frac{1}{2 R} \lambda_{a}$, the gauge transformations of $H_{a}$ and $\phi$ are

$$
\begin{aligned}
\delta H_{a} & =\lambda_{a b} H^{b}+\frac{1}{2 R} \lambda_{a} \phi, \\
\delta \phi & =-\frac{1}{2 R} \lambda_{a} H^{a} .
\end{aligned}
$$

Thus, in the limit $R \rightarrow \infty$ the fields $H_{a}$ and $\phi$ remain in the action as spin one and spin zero fields, but they decouple in the transformations and become independent.

When our $S O(1, d)$ gauge invariant gravitational action is taken in three dimensions, it is natural to ask whether the action obtained is identical to the Chern-Simons action which was also shown by Achucarro-Townsend [15] and Witten [16] to be equivalent to the Einstein action in three dimensions, but with a cosmological constant. In the Chern-Simons construction one uses only the gauge field $\omega_{\mu}^{A B}$ where the CS action is

$$
I_{\mathrm{CS}}=\frac{1}{2} \int d^{3} x \epsilon^{\mu \nu \rho} \epsilon_{A B C D}\left(\omega_{\mu}^{A B} \partial_{\nu} \omega_{\rho}^{C D}+\frac{2}{3} \omega_{\mu}^{A B} \omega_{\nu}^{C E} \omega_{\rho E}^{D}\right) .
$$

Using the same decomposition for $\omega_{\mu}^{A B}$ as before, we get

$$
\frac{1}{R} \int d^{3} x \epsilon^{\mu \nu \rho} \epsilon_{a b c} b_{\mu}^{a}\left(\partial_{\nu} \omega_{\rho}^{b c}+\omega_{\nu}^{b e} \omega_{\rho e}^{c}-\frac{1}{12 R^{2}} b_{\nu}^{b} b_{\rho}^{c}\right)
$$

which is the the first order formulation of the Einstein action plus a cosmological constant, with the dreibein field $b_{\mu}^{a}$. The special case with the $I S O(1, d-1)$ gauge group can be recovered by rescaling the action by $R$ and then taking the limit $R \rightarrow \infty$. In our treatment, there is also the additional field $e_{A}^{\mu}$ which is not a gauge field. The field $b_{\mu}^{a}$ is given by

$$
b_{\mu}^{a}=2 e_{\nu}^{a} \nabla_{\mu} c^{\nu}
$$

where $e_{\nu}^{a}$ is the inverse of $e_{a}^{\nu}$. Our action can be expressed in terms of $e_{a}^{\nu}$ and a nonpropagating field $c^{\mu}$. Comparing the two formulations, we deduce that the field $b_{\mu}^{a}$ must be identified with $e_{\mu}^{a}$. Although $e_{\mu}^{a}$ is not a gauge field, it can be shown, using the torsion constraint, that its diffeomorphism transformation with parameters $\zeta^{\mu}$ can yield the same gauge transformation as $b_{\mu}^{a}$ with the gauge parameter $\lambda^{a}=e_{\mu}^{a} \zeta^{\mu}[16]$. It then clear that although both formulations have the same gauge symmetry, they have different field configurations. Moreover, the usual matter couplings in the CS formulation are not possible because the dreibein $b_{\mu}^{a}$ is a gauge field. Any direct coupling to matter breaks gauge invariance, except for coupling to Wilson lines. In our case since $e_{A}^{\mu}$ is not a gauge field, a gauge invariant metric can be easily formed $g^{\mu \nu}=e_{A}^{\mu} e^{\nu A}$ and coupled to any form of matter desired.

\section{Acknowledgments}

The work of AHC is supported in part by the Alexander von Humboldt Foundation and by the National Science Foundation 0854779. V.M. is supported by TRR 33 "The Dark Universe" and the Cluster of Excellence EXC 153 "Origin and Structure of the Universe". 
Open Access. This article is distributed under the terms of the Creative Commons Attribution Noncommercial License which permits any noncommercial use, distribution, and reproduction in any medium, provided the original author(s) and source are credited.

\section{References}

[1] R. Utiyama, Invariant theoretical interpretation of interaction, Phys. Rev. 101 (1956) 1597 [SPIRES].

[2] T.W.B. Kibble, Lorentz invariance and the gravitational field, J. Math. Phys. 2 (1961) 212 [SPIRES].

[3] S. Weinberg, Generalized theories of gravity and supergravity in higher dimensions, in Proceeding of fifth workshop on grand unification, Editors K. Kang, H. Fried and P. Frampton, World Scientific (1984).

[4] C. Misner, K. Thorne and J. Wheeler, Gravitation, W.H. Freeman and Company (1973).

[5] A.H. Chamseddine and P. West, Supergravity as a gauge theory of supersymmetry, Nucl. Phys. B129 (1977) 39.

[6] S. MacDowell and F. Mansouri, Unified geometric theory of gravity and supergravity, Phys. Rev. Lett. 38 (1977) 739.

[7] J. Scherk, Extended supersymmetry and extended supergravity theories, in recent developments in gravitation, Cargese 1978, Editors M. Levy and S. Deser, Plenum Press (1978).

[8] E. Cremmer, Supergravities in five dimensions, Proceedings Nuffield workshop on superspace and supergravities, editors M. Rocek and S. Hawking, Cambridge University Press, Cambridge U.K. (1981).

[9] A.H. Chamseddine and H. Nicolai, Coupling the SO(2) supergravity through dimensional reduction, Phys. Lett. B 96 (1980) 89 [SPIRES].

[10] A. Einstein, A generalization of the relativistic theory of gravitation, Annals Math. 46 (1945) 578 [SPIRES].

[11] A. Einstein and E.G. Strauss, A generalization of the relativistic theory of gravitation. 2, Annals Math. 47 (1946) 731 [SPIRES].

[12] T. Damour, S. Deser and J.G. McCarthy, Nonsymmetric gravity theories: Inconsistencies and a cure, Phys. Rev. D 47 (1993) 1541 [gr-qc/9207003] [SPIRES].

[13] A.H. Chamseddine, Complexified gravity in noncommutative spaces, Commun. Math. Phys. 218 (2001) 283 [hep-th/0005222] [SPIRES].

[14] A.H. Chamseddine and V. Mukhanov, in preparation.

[15] A. Achucarro and P.K. Townsend, A Chern-Simons Action for Three-Dimensional anti-de Sitter Supergravity Theories, Phys. Lett. B 180 (1986) 89 [SPIRES].

[16] E. Witten, (2+1)-Dimensional Gravity as an Exactly Soluble System, Nucl. Phys. B 311 (1988) 46 [SPIRES]. 